\title{
Enhanced winnings in a mixed-ability population playing a minority game
}

\author{
N.F. Johnson ${ }^{a}$, P.M. Hui ${ }^{b}$, Dafang Zheng ${ }^{c}$, and M. Hart ${ }^{a}$ \\ a Physics Department, Clarendon Laboratory, Oxford University, Oxford OX1 3PU, U.K. \\ ${ }^{b}$ Department of Physics, The Chinese University of Hong Kong, Shatin, \\ New Territories, Hong Kong \\ ${ }^{c}$ Department of Applied Physics, South China University of Technology, \\ Guangzhou 510641, P.R. China
}

\begin{abstract}
We study a mixed population of adaptive agents with small and large memories, competing in a minority game. If the agents are sufficiently adaptive, we find that the average winnings per agent can exceed that obtainable in the corresponding pure populations. In contrast to the pure population, the average success rate of the large-memory agents can be greater than 50 percent. The present results are not reproduced if the agents are fed a random history, thereby demonstrating the importance of memory in this system.
\end{abstract}

PACS Nos.: 05.65.+b, 01.75.+m, 02.50.Le, 05.40.+j 
The subject of emergent phenomena in complex adaptive systems is attracting much interest among workers in physics, economics, biological and social sciences [1]. The minority game, introduced by Challet and Zhang [2] and studied subsequently by various workers [3] [7, represents a fascinating toy-model of a dynamical, complex adaptive system where members of the population (agents) repeatedly compete to be in a minority. It offers, for example, a simple paradigm for the decision dynamics underlying financial markets: more buyers than sellers implies higher prices, hence it can be better for a trader to be in the minority group of sellers. Subsequent to Challet and Zhang's pioneering work [2], studies of the minority game have focused on a 'pure' population where all agents have the same memory, or brain-size, $m$. A particularly interesting observation by Savit [3] is that while correlations can arise in the resulting (endogenous) history time-series for the pure population, these correlations cannot be further exploited for profit by the agents themselves.

In this paper, we explore the performance of a mixed-ability population where individual agents have either a small or large memory. If the agents are sufficiently adaptive, we find that the average winnings per agent can exceed that obtainable in the corresponding pure populations, achieving a maximum at a non-zero mixing ratio. In contrast to the pure population, the average success rate of the large-memory agents can exceed 50\%. These results, however, are not reproduced if the agents are fed a random history, thereby demonstrating the importance of the true history (and hence memory) in this system.

The minority game [2 [7] takes the form of a repeated game with an odd number of agents $N$ who must choose independently whether to be in room ' 0 ' or room ' 1 '. The winners are those in the room with fewer agents. The 'output' is a single binary digit, 0 or 1 , representing the winning room for each time step. This output is made available to all agents, and is the only information they can use to make decisions in subsequent turns. The memory $m$ is the length of the recent history bit-string that an agent can use when making its next decision [2] 5, 77. We consider a population containing $n_{m}$ agents with memory $m$, and $n_{m^{\prime}}=N-n_{m}$ agents with memory $m^{\prime}$. Consider $m=3$; there are $2^{2^{m}}=256$ possible strategies, each of 
which can be represented by a string of 8 bits ( 0 or 1 ) corresponding to the decisions based on the $2^{3}=8$ possible histories 000,001 etc. . The agents randomly pick $s$ strategies at the beginning of the game, with repetitions allowed. After each turn, the agent assigns one (virtual) point to each of his strategies which would have predicted the correct outcome. In addition the agent gets awarded one (real) point if he is successful. At each turn of the game, the agent uses the most successful strategy (i.e. most virtual points) from his bag of $s$ strategies. The success of any particular strategy is generally short-lived. If all the agents begin to use similar strategies, such a strategy ceases to be profitable and is dropped. Hence there is no best strategy for all times.

There are two 'spaces' of interest in the minority game, both of which depend on $m$ : (a) Strategy space. This forms a $2^{m}$-dimensional hypercube for memory $m$ with strategies at the $2^{2^{m}}$ vertices [2]. If the size of the strategy space is small compared to the total number of agents $N$ (i.e. $2.2^{m}<<N s$ [2] many agents may hold the highest-scoring strategy at any given turn and hence make the same decision. This leads to a large standard deviation in the winning room and hence a relatively low number of total points awarded [2 5, 7]. Such crowd-effects are a strategy-space phenomenon and have been shown to quantitatively explain the fluctuations for the pure population as a function of $m$ and $s$ [4]. Consider the average points per agent per turn $W$, defined as the total number of points awarded in that turn divided by the total number of agents. For small $m, W$ is substantially less than 0.5 due to the crowd-effects mentioned above. The maximum possible $W$ would correspond to the winning-room attendance permanently staying at $(N-1) / 2$. Therefore $W$ is always less than or equal to $(N-1) / 2 N$, hence $W<0.5$. Note that an external (i.e. non-participating) gambler using a coin-toss to predict the winning room, would have a $50 \%$ success rate since he would not suffer from this intrinsic crowding in strategy-space.

(b) History space. This forms an $m$-dimensional hypercube whose $2^{m}$ vertices correspond to all possible recent history bit-strings of length $m(000,001$ etc. for $m=3)$. For a pure population of agents with memory $m$, where $2.2^{m}<<N s$, there is information left in the history time-series [3]: however this information is hidden in bit-strings of length greater 
than $m$ and hence is not accessible to the agents [3]. For large $m$, there is information left in bit-strings of any length: however the agents have insufficient strategies to further exploit this information [3]. Cavagna has shown [6] that the standard deviation of the attendance of a given room (e.g. room 0) is changed little if the real history is replaced by a random one at each turn of the game: this follows from the fact that the agents cannot exploit any remaining correlations in the real history time-series.

Figure 1 shows the average winnings per agent per turn $W$ for a mixed population of $N=101$ players, with memory $m=3$ or $m^{\prime}=6$, obtained using numerical simulations. The number of $m=3$ agents $n_{3}$ is shown on the x-axis, hence the number of $m^{\prime}=6$ agents is given by $n_{6}=101-n_{3}$. Each agent has $s=7$ strategies. The parameters are chosen so that the pure populations lie in the crowded, or frustrated, regime (i.e. $2.2^{m}<<N s$ ) since we are interested in investigating whether our mixed population can adapt to relieve this frustration. Each data-point corresponds to an average over 32 runs, with data being collected in the limit of long times. For clarity the spread in values at each data-point is not shown; these spreads are sufficiently small for us to be certain that the features we discuss here are not numerical artifacts. For the 'real history' results, the $m=3\left(m^{\prime}=6\right)$ agents are supplied with the last 3 (6) bits of the winning-room time-series. For the 'random history' results, a random 6-bit string is generated at every turn of the game and given to the $m^{\prime}=6$ agents; the $m=3$ agents are only given the last 3 digits of this string. A striking result of Fig. 1 is that the average number of points per agent $W$ (solid circles) shows a maximum at finite mixing, i.e. at $n_{3} \sim 35, n_{6} \sim 65$. The total number of points awarded per turn can therefore exceed that corresponding to either pure $m=3$ or pure $m^{\prime}=6$ populations. This result is not reproduced if the history supplied to the agents is random (open circles).

Figure 2 shows the average winnings per turn for the $m=3$ and $m^{\prime}=6$ agents separately. The straight line at 0.5 corresponds to the average success-rate for a non-participating agent predicting winning rooms using a coin-toss; as discussed above, it is also the upper limit for the average winnings per agent in a pure population. For $n_{3}>45$, the average $m^{\prime}=6$ agent (solid triangles) manages to beat the coin-toss agent and systematically profit from 
the game (see also Ref. [2]). This cannot occur in the minority game with pure populations, regardless of the memory $m$. Although certain agents in the pure population may manage to beat the coin-toss if the strategy space is sufficiently large, the average winnings per agent is still less than 0.5. It is surprising that this systematic profiteering by $m^{\prime}=6$ agents in the mixed population can occur up to relatively large values of $n_{6}$, i.e. in populations of up to $\sim 55 \% m^{\prime}=6$ players. Although the random-history result reproduces the $m=3$ agents' winnings fairly well (open squares), it is quite inaccurate for the $m^{\prime}=6$ agents: specifically, the $m^{\prime}=6$ players in the random-history game (open triangles) always do worse than the coin-toss. This is essentially because the $m^{\prime}=6$ agents in the real-history game have the opportunity to exploit correlations in the real-history time-series left by the $m=3$ agents. The enhanced average winnings for $m^{\prime}=6$ agents in Fig. 2, together with the corresponding peak in Fig. 1, result from the subtle interplay of history-space and strategy-space effects and demonstrate the importance of the memory in this system.

Figure 2 shows that as $n_{3} \rightarrow 1$, and hence $n_{6} \rightarrow N-1$, the remaining $m=3$ agents manage to increase their average winnings. In fact a single $m=3$ agent in an $m^{\prime}=6$ population wins the same amount as the average $m^{\prime}=6$ agent. This surprising result can be understood by realizing that for $n_{6} \rightarrow N$, the $m^{\prime}=6$ population is in the 'crowd' region [2 [4], hence the $m^{\prime}=6$ agents win a relatively low number of points on average. For $n_{3}>60$, the average winnings per $m=3$ agent saturates at the value corresponding to a pure $m=3$ population, despite the fact that the $m^{\prime}=6$ agents are still systematically profiting from the game. In this sense, our competitive agents are also demonstrating some degree of (unintentional) mutual cooperation.

As the number of strategies per agent $s$ decreases, for fixed $m=3$ and $m^{\prime}=6$, the peak in Fig. 1 becomes less pronounced. For $s=2$, there is no discernible peak. This suggests that the benefits of a mixed population, in terms of maximizing the total number of points awarded, may require a certain level of adaptivity. Even at $s=2$, however, the average winnings of the large-memory $m^{\prime}=6$ agents is still greater than 0.5 in the limit of small $n_{6}$. Note that the main results presented here do not vary significantly if $N$ is increased. 
Detailed results as a function of $m, m^{\prime}, s$ and $N$ will be presented elsewhere.

We now discuss the reasons why the real and random histories might produce different results. Consider the $2^{6}=64$ possible histories for $m^{\prime}=6$. Suppose the system has reached ...001010 at time $t$. The subsequent evolution on the 6-dimensional history-space hypercube allows a number of possible trajectories starting at the vertex 001010. For example, at time $t+1$ the system can be at 010100 or 010101 ; however it cannot be at any other of the 64 possible vertices. We therefore see that the $m^{\prime}=6$ agents pass through the history-space, and hence update their strategies, by moving on a particular subset of all possible trajectories. In contrast, the random-history model allows the system to jump to any history-space vertex with equal probability at a given time-step. If the game only contains a pure population, the agents cannot access any additional correlations in the real-history time-series [3]: it therefore matters little if the system visits all history-space vertices in a deterministic or random way. The random-history and real-history results are therefore similar for pure populations (see Fig. 1 and Ref. [6]). However when the two sets of agents coexist, there are two separate time-scales for passing through the respective history-spaces, i.e. $\tau_{6}>2^{6}=64$ for $m^{\prime}=6$ agents and $\tau_{3}>2^{3}=8$ for $m=3$ agents. The time-scale $\tau_{3}$ for $m=3$ agents will be relatively short, hence random-history and real-history models should provide similar results for $m=3$ agents, as demonstrated in Fig. 2. On this $\tau_{3}$ time-scale, however, the $m^{\prime}=6$ real-history agents are dynamically updating their strategies in a qualitatively different way from the $m^{\prime}=6$ random-history agents, because of this distinction between real-history and random-history trajectories. The $m^{\prime}=6$ real-history agents now have the opportunity to exploit specific correlations in the real-history time-series left by the $m=3$ agents.

Fluctuations in the winning room attendance away from $(N-1) / 2=50$ imply wasteage of total points [2]. Hence $W \sim 0.5-\frac{\sigma}{N}$, where $\sigma$ is the standard deviation of the attendance in one of the rooms, say room 0 . For a pure population in the crowded regime, $\sigma \propto N$ : hence we write $\sigma \sim C N$ where $C$ is a constant of proportionality (see Ref. [4] for a more detailed analytic analysis). This expression includes the crowd-effect in strategy-space involving 
agents with equal memory 4 . For the mixed population, we assume that the corresponding $\sigma$ is obtained by adding separately the contributions to the variance from the $m=3$ agents and the $m^{\prime}=6$ agents; defining the concentration of $m=3$ agents as $x=\frac{n_{3}}{N}$, then $\sigma^{2} \sim \sigma_{3}^{2}+\sigma_{6}^{2}$ where $\sigma_{3} \sim C_{3} x N$ and $\sigma_{6} \sim C_{6}(1-x) N$. This latter assumption of additive variances assumes that the system has managed to remove any internal frustration between agents with different memories, i.e. the two groups of agents behave independently. This is not unreasonable given the interplay between history and strategy-spaces in the real-history model. Hence in the mixed population $W \sim 0.5-\left[C_{3}^{2} x^{2}+C_{6}^{2}(1-x)^{2}\right]^{1 / 2}$. For $x \rightarrow 0$ we obtain a straight line $W \sim 0.5-C_{6}+C_{6} x$ with positive slope (consistent with real-history results in Fig. 1), while for $x \rightarrow 1$ we obtain a straight line $W \sim 0.5-C_{3} x$ with negative slope (again consistent with Fig. 1). This theoretical curve does exhibit a maximum at $x \sim 0.18, W \sim 0.428$. Intriguingly, however, it tends to underestimate the real-history winnings suggesting that the actual population is exhibiting an additional degree of cooperation (correlation). A fuller theory of this mixed system is therefore needed which will incorporate such additional inter-agent correlations.

In summary, we have studied the performance of a binary population containing agents with dissimilar memories. Both the strategy-space ('configurational') and history-space ('temporal') correlations play a crucial role in the system's evolution. The importance of memory has been demonstrated. The present results may contribute to a better understanding of optimization in generic multi-agent systems where resources are limited, e.g. social dilemmas and Internet congestion 8-10. Assuming that the present system provides a simple model of a marketplace, our results suggest that a surprisingly large number of 'superior' $\left(m^{\prime}=6\right)$ traders can consistently 'beat the market' by uncovering and exploiting hidden correlations in the system's recent history without being directly detrimental to the remainder: needless to say that the usefulness of such technical analysis, or 'charting', is still a hotly debated issue in the financial world.

We thank A. Short for additional computer simulations, and D. Challet, D. Leonard, D. Sherrington and A. Cavagna for discussions regarding the pure-population minority game. 


\section{REFERENCES}

[1] See, for example, J.H. Holland, Emergence (Oxford University Press, Oxford, 1998).

[2] D. Challet and Y.C. Zhang, Physica A 246, 407 (1997); Physica A 256, 514 (1998). These papers also include a discussion of particular mixed populations: however our present model and results have not appeared before.

[3] R. Savit, R. Manuca and R. Riolo, adap-org/9712006; R. Manuca, Y. Li, R. Riolo and R. Savit, adap-org/9811005.

[4] N.F. Johnson, M. Hart and P. M. Hui, Physica A (in press). See also cond-mat/9811227.

[5] M.A.R. de Cara, O. Pla and F. Guinea, cond-mat/9811162.

[6] A. Cavagna, cond-mat/9812215.

[7] R. D'hulst and G.J. Rodgers, adap-org/9902001.

[8] B.A. Huberman and R.M. Lukose, Science 277, 535 (1997).

[9] W.B. Arthur, Amer. Econ. Assoc. Papers and Proc. 84, 406 (1994).

[10] N.F. Johnson, S. Jarvis, R. Jonson, P. Cheung, Y.R. Kwong and P.M. Hui, Physica A 256, 230 (1998). 


\section{Figure Captions}

Figure 1: Average winnings per agent per turn $W$ for a mixed population of $N=101$ players, with memory $m=3$ or $m^{\prime}=6$, obtained numerically. Each agent has $s=7$ strategies. Each data-point corresponds to an average over 32 runs. See text for an explanation of 'real history' and 'random history'.

Figure 2: Average winnings per turn for $m=3$ and $m^{\prime}=6$ agents separately. Other parameters are as in Figure 1. See text for an explanation of 'coin-toss'. 
Fig. 1 [Johnson et al. "Enhanced winnings..."]

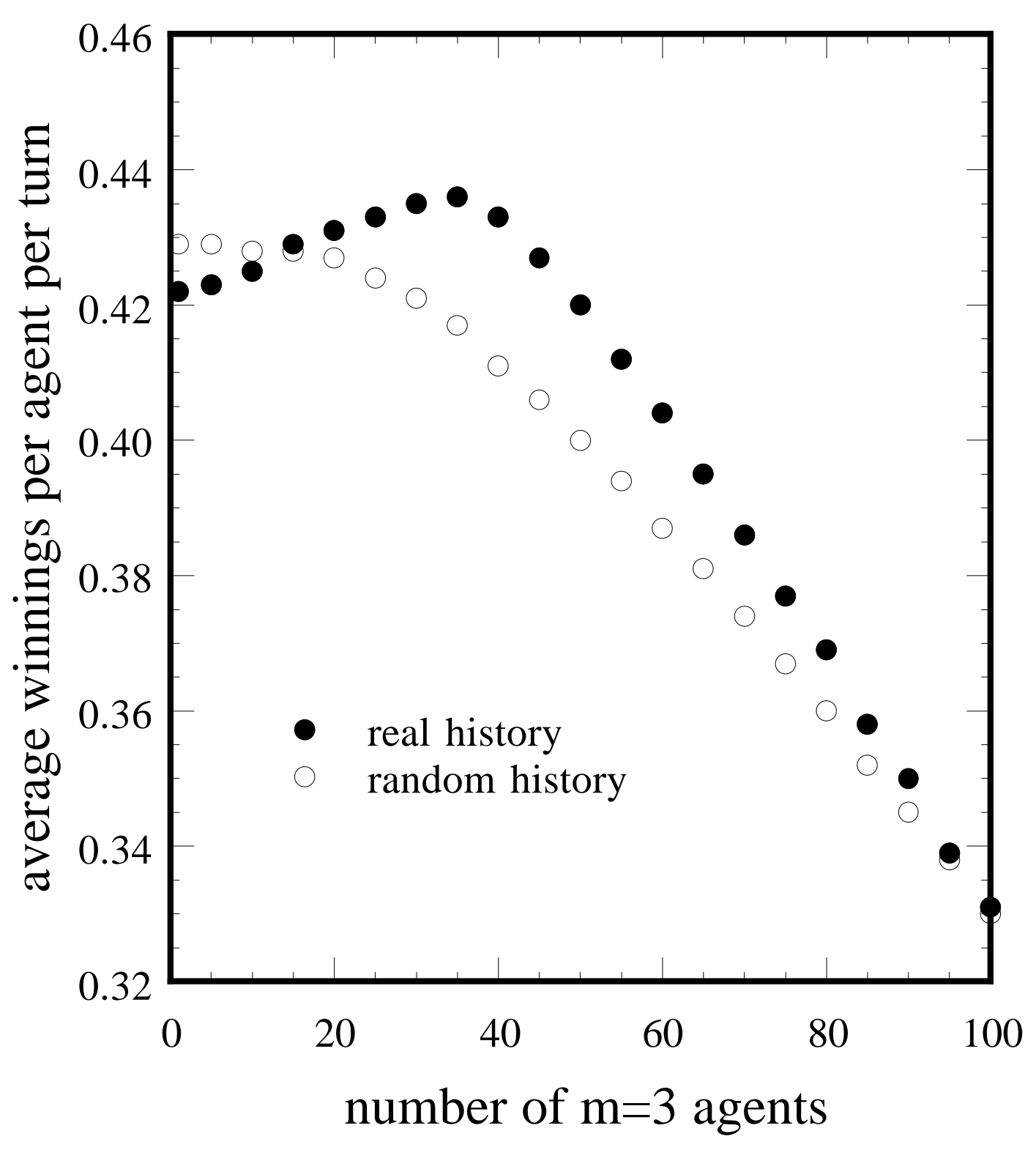


Fig. 2 [Johnson et al. "Enhanced winnings..."]

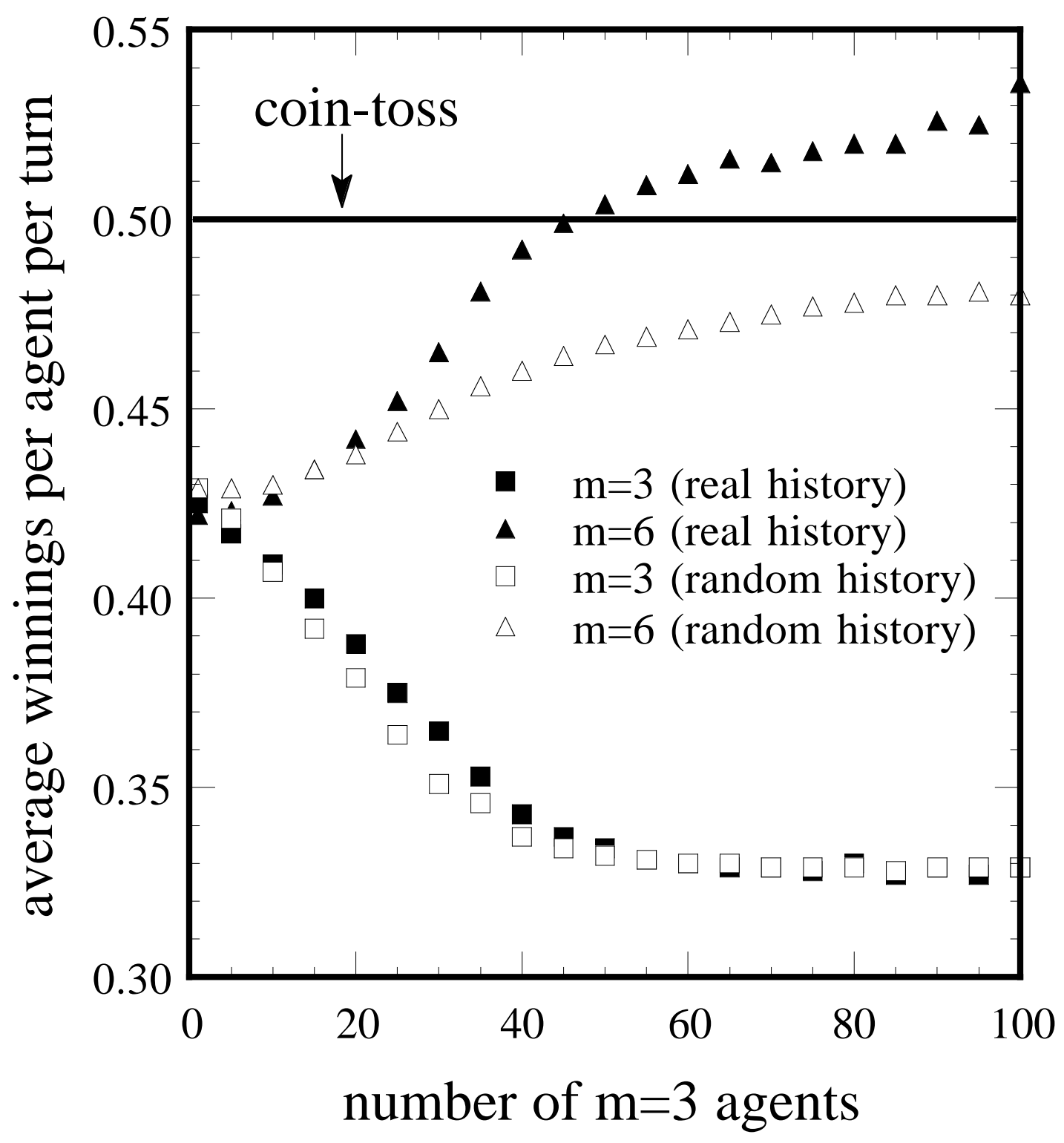

